\title{
Performance assessment of a vertical axis turbine in a marine current flume tank and CFD modelling
}

\author{
Rolland S.A. ${ }^{1,{ }^{*}}$, Thatcher M. ${ }^{2}$, Ellis R. ${ }^{3}$, Gaurier Benoit ${ }^{4}$, Croft T.N. ${ }^{1}$, Cross M. ${ }^{1}$ \\ ${ }^{1}$ Swansea University, College of Engineering, SA2 8PP, Swansea, UK \\ ${ }^{2}$ Quadratec Ltd, NP26 5PS, Caldicot, UK \\ ${ }^{3}$ C-FEC Ltd, Digital Technium, SA2 8PP, Swansea, UK \\ ${ }^{4}$ IFREMER, 150, Quai Gambetta - 62200 Boulogne-sur-Mer, France \\ * Corresponding author: S. A. Rolland, Tel.: +44(0)1792606871; \\ email address : $\underline{\text { s.rolland@swansea.ac.uk }}$
}

\begin{abstract}
:
As advances in numerical modelling techniques support the increased confidence in predictions from computer simulations, the need remains to have experimental verification built into the design process. This paper outlines the experimental investigation carried out on a shielded vertical axis turbine in a marine environment. The experiments consist of performance measurements and the use of particle image velocimetry on a small scale device in a marine current flume. The results demonstrate that the performance of the device can be modelled numerically; in particular, the results show that the numerical model used can correctly predict the increase in performance with Reynolds number.
\end{abstract}

\section{Highlights}

- Identification of the energy conversion mechanisms in a vertical axis turbine. Validation of a CFD model with experimental performance data and PIV. Turbine performance increases with Reynolds number due to changes in flow regime.

Keywords : Computational fluid dynamics, Cross-flow turbine, Flume test, Power coefficient, Model validation 


\section{Introduction}

The attention given to marine energy sources is increasing as demonstrated by the interest from major manufacturers and the allocation of national resources to the development of new energy recovery devices [1]. This study presents a set of experiments designed to verify numerical simulations of a shielded vertical axis turbine in water. The test series seeks to answer the questions raised at the small scale tank testing stage of the concept appraisal process laid out by Johnstone et al. [2]. The present work addresses several of the questions raised at the small scale testing: the proof of concept is implicitly addressed, of greater import, the CFD (Computational Fluid Dynamics) model validation is ultimately the objective of this test series. This is due to the nature of the development cycle of the turbine, which relies principally on CFD modelling for cost effectiveness, with milestone experiments integrated in the process to validate the model results.

The numerical approach to the CFD modelling has been described and verified for wind turbine devices [3] [4]. The motivation for further experimental work is the change of environment combined with the simplified approach to turbulence modelling. Prior work by Bachant and Wosnik demonstrated that that the performance of cross flow energy converters is Reynolds number dependent [5]. The change in environment will lead to changes in Reynolds number. The same publication also highlighted the difficulty in reflecting this dependency in numerical simulations. The simplified approach to turbulence modelling proposed by the authors is closely linked to the Reynolds dependency validation. Much effort has been undertaken in numerical modelling to predict the properties of turbines, focusing on turbulence modelling. Bachant and Wosnik used a k-omega model, for example and found 
that the experimental data did not show as strong a Reynolds dependency as numerically predicted. The difficulty in modelling vertical axis wind turbine is the broad range of incident angles encountered by the blades. This difficulty is identified by Lanzafame et al. [6], who used the $k-\omega$ SST [7] and the Transition SST turbulence models. Their work shows that a good prediction of blade properties is achievable but requires a "long process of optimizing the local correlation variables", which is not fully described. McNaughton et al. [8] used a modification of the SST $k-\omega$ for low Reynolds number ( $k-\omega$ SST LRE). The $k-\omega$ SST based model over-predicted the power coefficient, while the $k-\omega$ SST LRE based model under-predicted the power coefficient. Moreover, the latter model resulted in discrepancies in the dynamic response that were not exhibited when using the $k-\omega$ SST model. Li et al. adopted the more sophisticated large eddy simulation (LES) approach and compared it to the traditional Reynolds average Navier-Stoke solutions [9]. The study showed an improvement of the stall behaviour prediction with the adoption of the LES technique and led the authors conclude that the dynamic stall prediction is delayed by the use of RANS methods. The lack of a satisfactory consensus in the research community motivated the choice of a pragmatic approach to turbulence modelling, laid out in section 3 and supported by Rolland et al. [4].

The originality in the present work resides in the transient modelling of the turbine, which, due to the geometry does not readily lend itself to steady state models combined with the simplified turbulence modelling approach laid out in the numerical method section. This is in contrast with techniques such as the blade element model, for example, where the blade properties are inputs to the models [10] and it represents a further challenge which needs to be resolved. The study reports the experimental programme undertaken to support the CFD modelling approach used to assess the correlation observed between numerical and experimental results. The experimental work was carried out at the IFREMER flow tank, which has a published record of marine turbine studies, including flow characterisation by particle image velocimetry (PIV) [11] [12].

\section{Experimental Method}

A prototype of the proposed design (Figure 1) was built with a diameter of $0.5 \mathrm{~m},(1 / 8$ of flume width) and $0.5 \mathrm{~m}$ high (0.24 flume depth) with a disc above the rotor, $0.4 \mathrm{~m}$ below the water surface, to minimise the surface effects (Figure 1a). An immersion of one turbine diameter, also equal to the turbine's height can be considered sufficient to avoid surface effects. This is supported by the study by Bahaj et al., in which cavitation tunnel results and towing tank results showed little difference for an immersion of 0.55 turbine diameters [13]. The design region of interest in this study was the rotor and the shield outlined in Figure $1 \mathrm{~b}$. A stepper motor was used to regulate the rotation speed of the turbine. The instrumentation consisted of a shaft encoder to record the rotating speed and a torque transducer, measuring the torque output of the turbine. The torque from the turbine was corrected for friction with a $4 \mathrm{~N} \mathrm{~m}$ constant component and a variable component according to the bearing manufacturer guidelines based on estimated radial loads and a journal bearing friction coefficient of 0.05 .

Experiments were carried out in the IFREMER (French Institute for the Exploitation of the Sea) wave and current circulating tank displayed in Figure 2. The flume is $18 \mathrm{~m}$ long by $4 \mathrm{~m}$ wide and $2 \mathrm{~m}$ deep. A side observation window of $8 \mathrm{~m}$ by $2 \mathrm{~m}$ placed on one side of the tank allows users to observe the behaviour of the models during trials. The turbulence level can be adjusted between $3 \%$ and $15 \%$, by the modification of grids and flow straighteners at the entry of the test section, in order to simulate more realistic flows. The stream-wise flow velocity can be adjusted between 0.1 and $2.2 \mathrm{~m} / \mathrm{s}$. The experiments were carried out at flow 
speeds of $0.8 \mathrm{~m} / \mathrm{s}, 1 \mathrm{~m} / \mathrm{s}, 1.2 \mathrm{~m} / \mathrm{s}$ and $1.4 \mathrm{~m} / \mathrm{s}$. At each flow speed they were repeated at tip speed ratios (TSRs) from 0.0 to 0.7 in increments of 0.1 . Particle image velocimetry measurements were taken at flow speeds of $0.8 \mathrm{~m} / \mathrm{s}$ and tip speed ratios of $0.0,0.3$ and 0.5 . An extensive description of the facility was given by Bouhoubeiny et al. [14].

a.

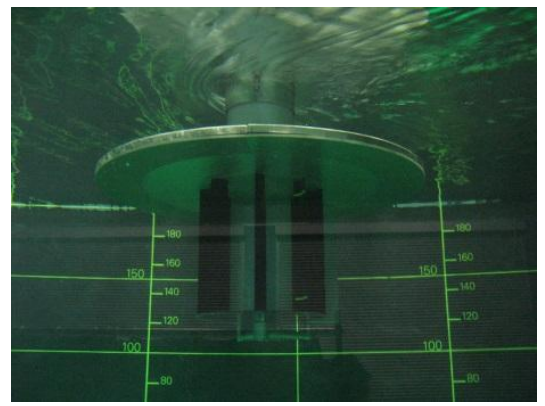

Flow direction

b.

Figure 1: Outline of the turbine in the tank and cross section.
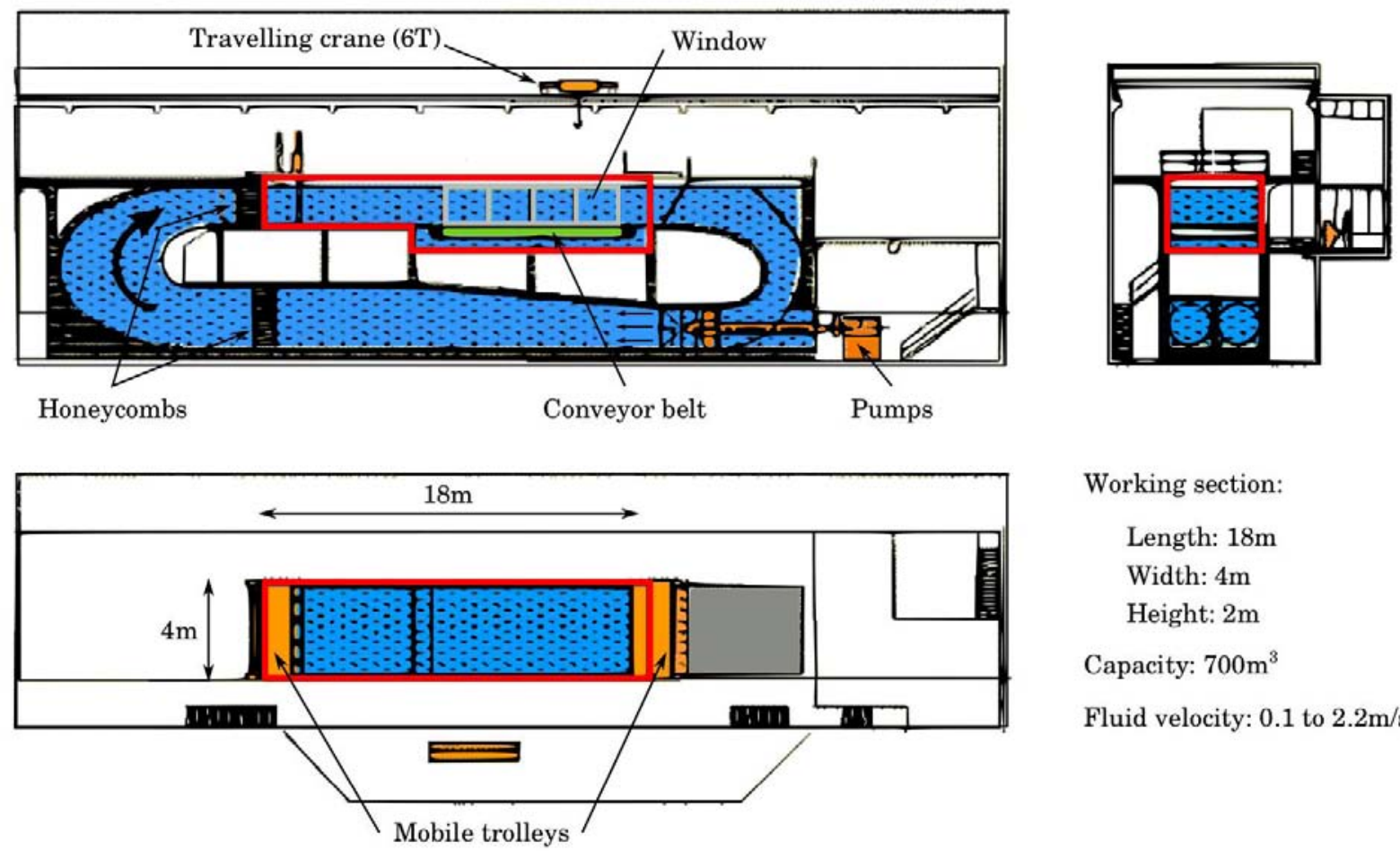

Working section:

Length: $18 \mathrm{~m}$

Width: $4 \mathrm{~m}$

Height: $2 \mathrm{~m}$

Capacity: $700 \mathrm{~m}^{3}$

Fluid velocity: 0.1 to $2.2 \mathrm{~m} / \mathrm{s}$

Figure 2: Schematic representation of IFREMER circulating tank

In this study, two-dimensional time-resolved PIV measurements were carried out (Figure 3). Illumination was provided by a standard, frequency-doubled, double-cavity Nd:YAG laser (NewWave, Gemini PIV) with a pulse energy of up to $120 \mathrm{~mJ}$ per pulse at $532 \mathrm{~nm}$ and maximum sample frequency of $15 \mathrm{~Hz}$. The seeding particles used for the experiments were silver-plated glass spheres of $15 \mu \mathrm{m}$ diameter. The image acquisition was carried out by a high resolution CCD camera with a $20 \mathrm{~mm}$ focal lens. The physical area of approximately $0.36 \mathrm{~m}$ by $0.27 \mathrm{~m}$ was captured onto 1600 by 1280 pixel $^{2}$ at a rate of $15 \mathrm{~Hz}$ and in synchronisation with the laser source. The FlowMap software from Dantec Dynamics was used for PIV image processing. Finally, instantaneous velocity fields were obtained using a cross-correlation PIV 
algorithm using $25 \%$ overlapped windows of $32 * 32$ pixels ${ }^{2}$. This yields $99 * 74$ instantaneous velocity vectors on a rectangular mesh of $357 * 268 \mathrm{~mm}^{2}$. The physical discretisation corresponds to a resolution of $3.6 \mathrm{~mm}$ in each direction and the time separation between two PIV images is $0.067 \mathrm{~s}$. The composite images were obtained by repositioning the camera and repeating the experiments, due to the field width required. The resulting images are presented as time averaged velocity contours or vectors.
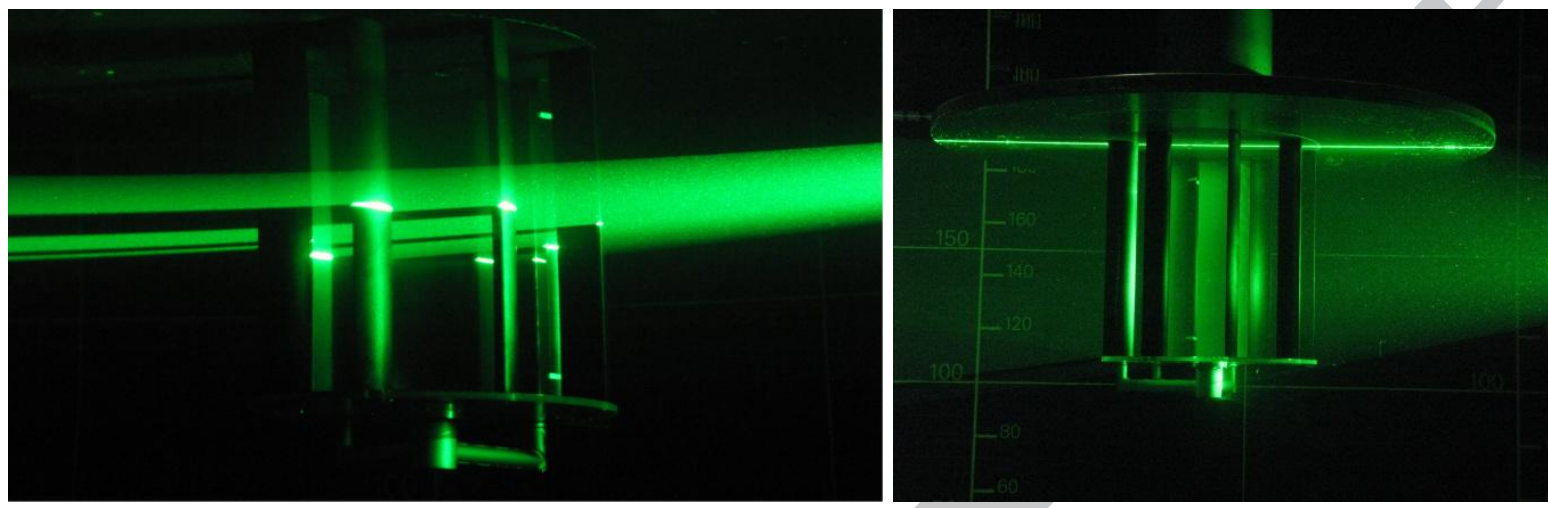

Figure 3: Horizontal and vertical planes PIV set-ups

\section{Numerical CFD Model}

The numerical modelling of the device was carried out using the research finite volume code PHYSICA, [15] to solve the transport equations for incompressible Newtonian flow:

$$
\begin{gathered}
\frac{\partial}{\partial t}(\rho \underline{u})+\nabla \cdot(\rho \underline{u u})=\nabla \cdot(\mu \nabla \underline{u})-\nabla p+\underline{S} \\
\rho \nabla \cdot(\underline{u})=0
\end{gathered}
$$

Here, $\rho, u, \mu$ and $p$ represent the density, velocity, viscosity and pressure of the fluid. $S$ represents a momentum source term. A cell-centred finite volume scheme is used to solve the transport equations over an unstructured mesh [16], using the Rhie-Chow interpolation for a smooth solution to the momentum equations [17] and the SIMPLEC scheme is used for pressure coupling [18] [19].

The flow is expected to be turbulent through the turbine. Turbulence modelling methods, reviewed in section 1, have been used with mitigated success. In particular, a need for calibration is highlighted in the literature reviewed [6]. A mesh independence study showed that with the use of a $k-\omega S S T$ turbulence model, grid independence can be achieved using 350,000 elements and 8 inflation layers near the wall, with the first layer at a wall distance $y^{+}=1$. The resulting power coefficients were reported over-predicted by a factor of 1.5 [20]. This led the authors to the simplest possible in capturing the effects of turbulence - an empirical approach to turbulence modelling was used, in which a value of viscosity for the fluid 200 times greater than the physical laminar viscosity and a laminar handling of the flow. This approach has been reported by Rolland et al. [4] and validated against experimental wind data [3]. The same factor of 200 was used in the present work on water turbines as was used in prior work [4]. The use of a laminar model implies that mesh independence can only be reached when direct numerical simulation is achieved, which is unrealistic at the scale of a whole turbine's model, which is why the use of a viscosity calibration case was necessary. 
The domain was discretised as a two-dimensional cross section of the turbine in a $4 \mathrm{~m}$ wide and $40 \mathrm{~m}$ long computational domain with $29.5 \mathrm{~m}$ downstream to avoid interference with outlet boundary conditions illustrated in Figure 4. An unstructured mesh was generated with 110,000 elements, $67 \%$ of which are located within the rotor, which was consistent with the calibration cases presented by Rolland et al., for use with the enhanced viscosity laminar model. The domain was split using a conforming sliding mesh technique illustrated in Figure 5. This enabled the transient modelling of the turbine while avoiding the use of deformed elements or an interpolation plane at the interface between the static and rotating subdomains. This is of particular importance as the asymmetry of the shield combined with the rotor motion implies that a steady state solution cannot be expected to exist.

The choice of a conforming sliding mesh technique couples the time step with the rotor speed for all tip speed ratios $\lambda \neq 0$. The resulting time steps are shown in Table 1 . It was found that convergence was more readily achieved by time stepping than by assigning a large number of iterations per time step, therefore, three complete revolutions were simulated for each case with 300 iterations per time step and the first two revolutions were not retained for the calculation of the power coefficient. For the cases where $\lambda=0$, a time step was assigned based on flow speed and 1440 time steps were carried out. Similarly to the cases where $\lambda \neq 0$, only the last 480 time steps were used to compute the results.
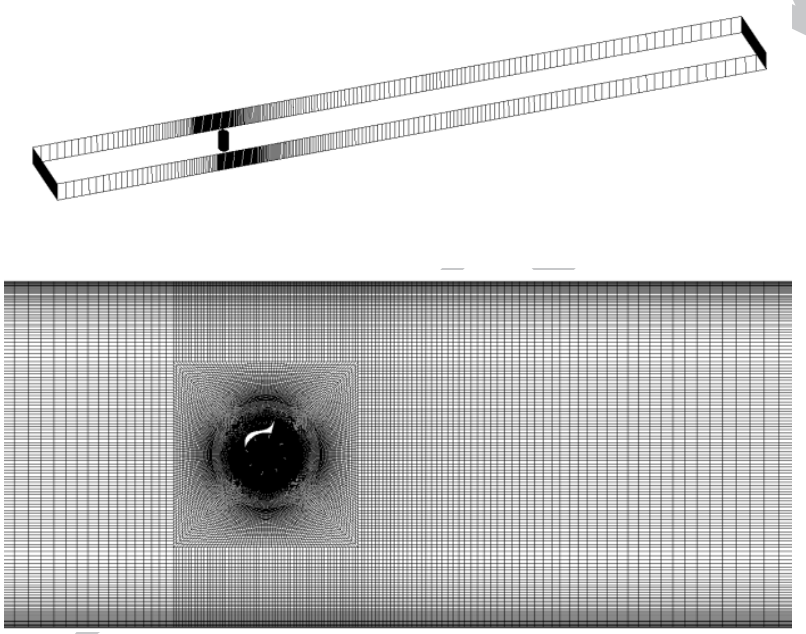

Figure 4: Computational domain layout and mesh density distribution around the turbine.

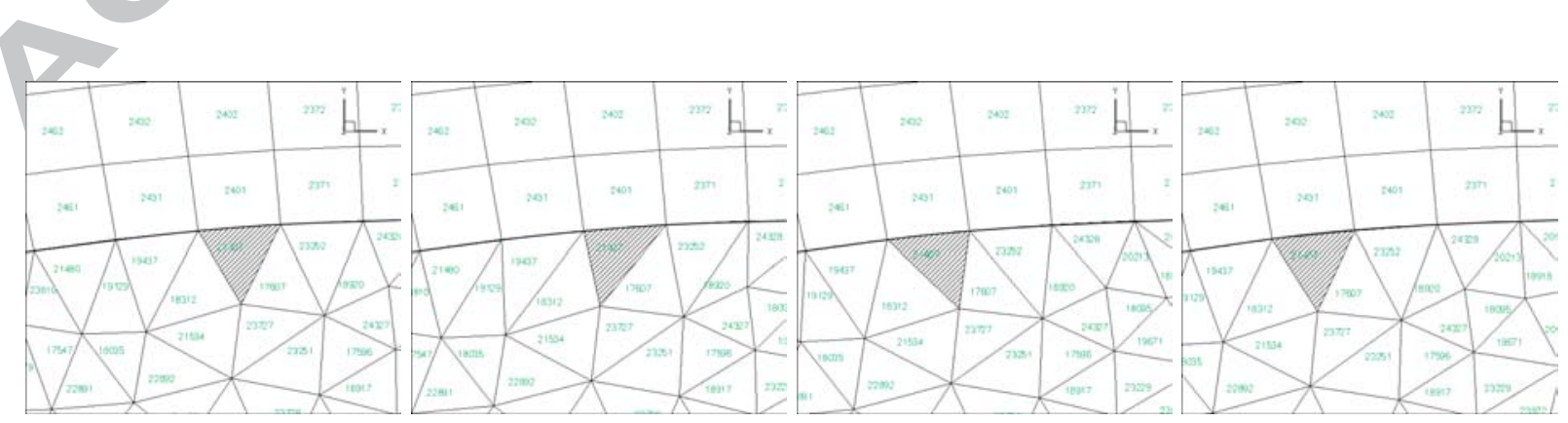

Figure 5: Illustration of the conforming sliding mesh technique. 
Table 1: time step sizes (s) used as a function of flow speed and TSR

\begin{tabular}{c|ccccccc} 
& $\lambda=0$ & $\lambda=0.1$ & $\lambda=0.2$ & $\lambda=0.3$ & $\lambda=0.4$ & $\lambda=0.5$ & $\lambda=0.6$ \\
\hline $0.8 \mathrm{~m} / \mathrm{s}$ & 0.520833 & 0.040906 & 0.020453 & 0.013635 & 0.010227 & 0.008181 & 0.006818 \\
$1.0 \mathrm{~m} / \mathrm{s}$ & 0.416667 & 0.032725 & 0.016362 & 0.010908 & 0.008181 & 0.006545 & 0.005454 \\
$1.2 \mathrm{~m} / \mathrm{s}$ & 0.347222 & 0.027271 & 0.013635 & 0.00909 & 0.006818 & 0.005454 & 0.004545 \\
$1.4 \mathrm{~m} / \mathrm{s}$ & 0.297619 & 0.023375 & 0.011687 & 0.007792 & 0.005844 & 0.004675 & 0.003896
\end{tabular}

\section{Results and Analysis}

The performance criterion used in this study is the power coefficient $(C p)$ scaled by the peak power coefficient experimentally measured $\left(C p_{\max }\right)$. This criterion is plotted in Figure 6 and shows that the numerical model is effective and accurate in its prediction of peak performance and the tip speed ratio at which it occurs (0.4). The main discrepancy occurs in the rise of the power coefficient with increasing tip speed ratio. Experimental results suggest a second order correlation between tip speed ratio and power coefficient whereas a near linear increase is numerically predicted. The curve fitting to the experimental results indicate that the tip speed ratio at which the maximum power coefficient is obtained may increase with the Reynolds number; however the quality of the curve fitting is too low to hold this as a conclusive result. In contrast, the tip speed ratio at which the maximum $C p$ is predicted numerically is essentially constant.

Notably, the increase of performance with increasing Reynolds number was predicted numerically and experimentally confirmed. This is a crucial result for future applications as larger scale turbines imply greater Reynolds number, thus offering a potential gain in power coefficient. With a Reynolds number based on the turbine diameter, the range explored is from $R e=3.06 \times 10^{5}$ to $R e=5.36 \times 10^{5}$. Figure 6 shows that a $75 \%$ increase in Reynolds number results in a $40 \%$ relative increase in power coefficient.

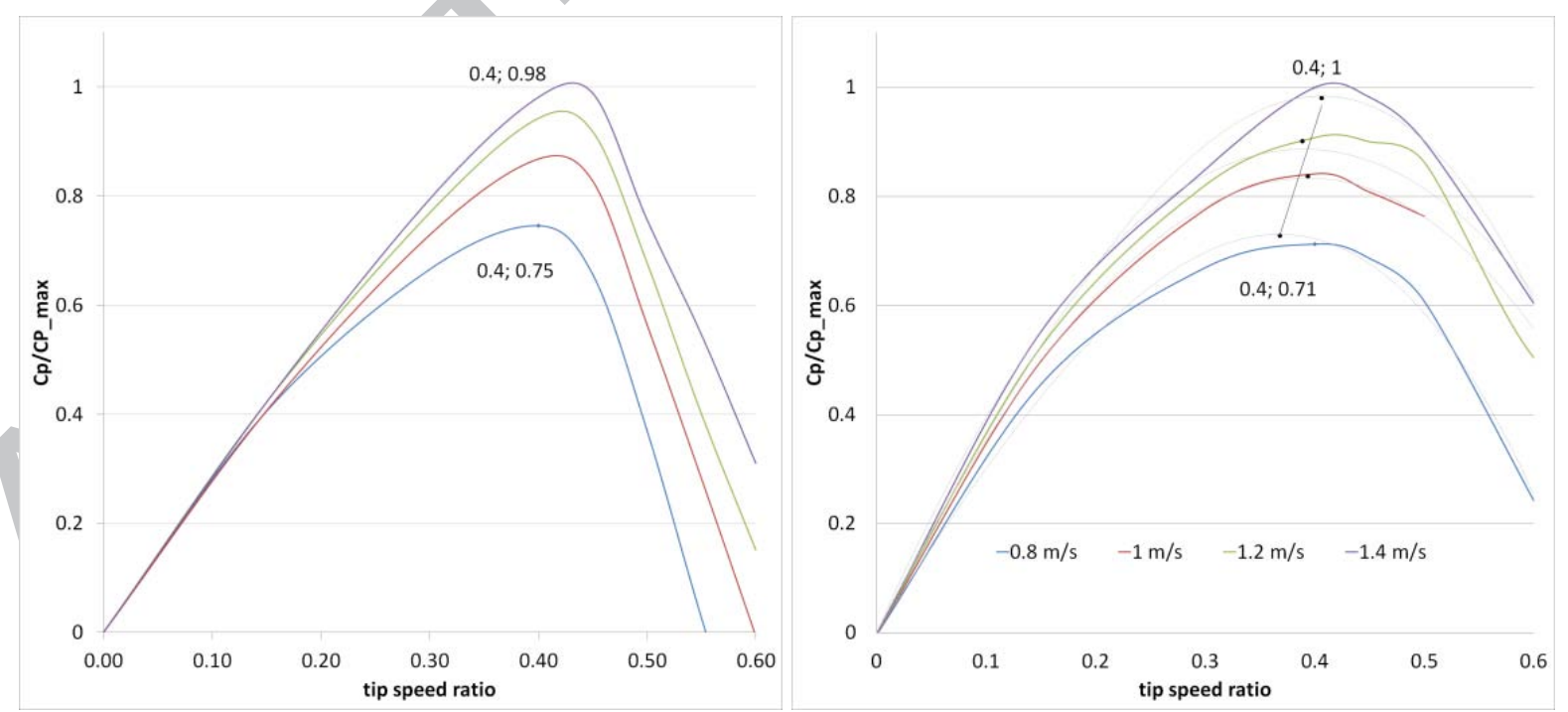

Figure 6: Comparison of numerically predicted $C p / C p_{\max }$ (left) and experimental data (right).

Two results were sought from the particle image velocimetry: an assessment of the 2D modelling strategy and a qualitative correlation between the flow fields predicted and observed. Figure 7 shows the location of the vertical PIV measurement plane in a vertical cross section of the turbine containing the turbine's axis. The flow in the tank is left to right in 
the images and the top disc is located at the top of the figure (arbitrarily $1800 \mathrm{~mm}$ ). The shadow of the turbine's components obscures some areas but the required images were captured. Processing the resulting velocity vectors, in Figure 8, shows that the effect of the tip speed ratio on the flow avoidance is negligible and $6 \%$ of the flow from $0.2 \mathrm{~m}$ upstream in the median plane avoids the turbine.

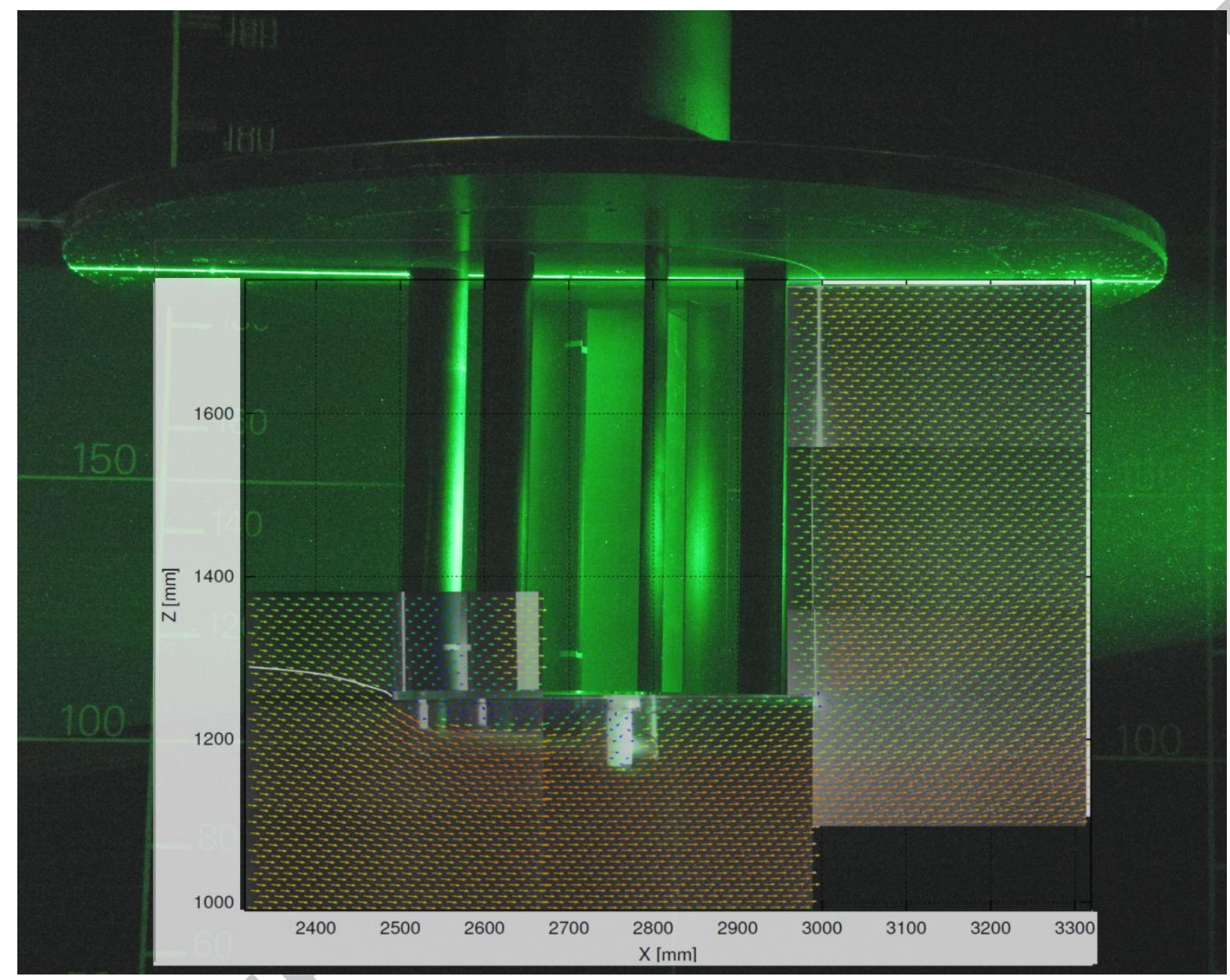

Figure 7: Photography and superimposed velocity vectors from the PIV for location reference. 


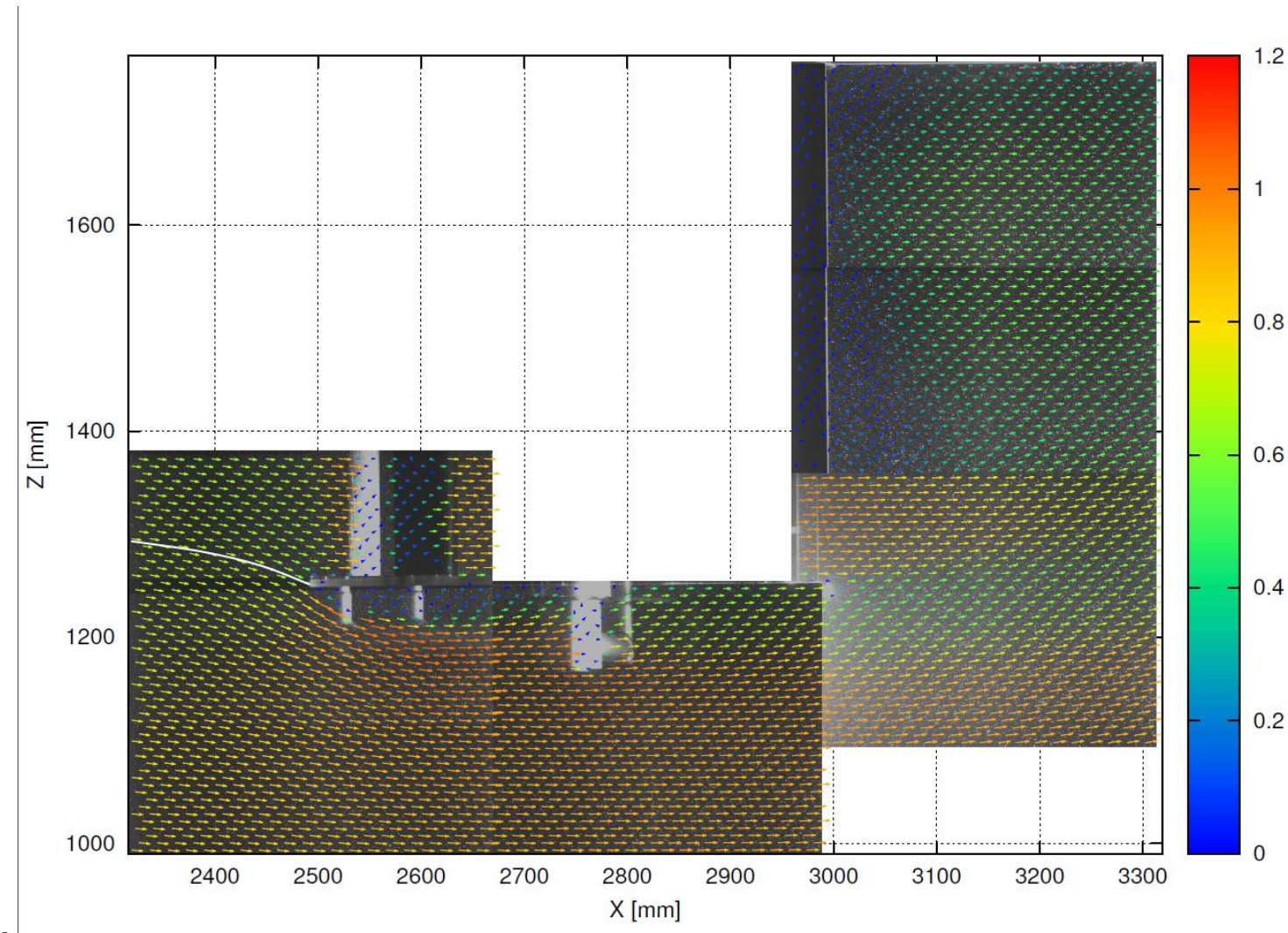

a.

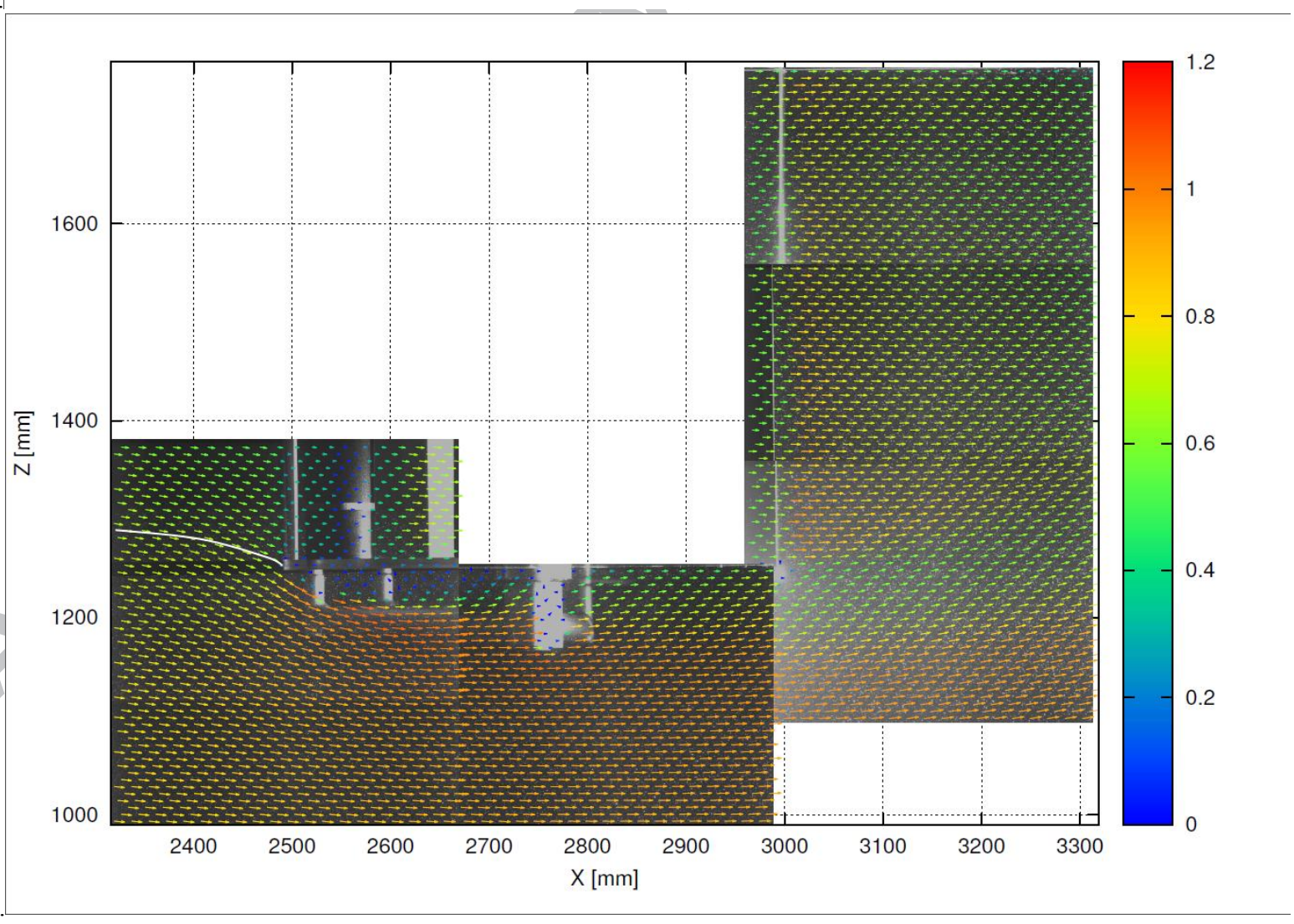




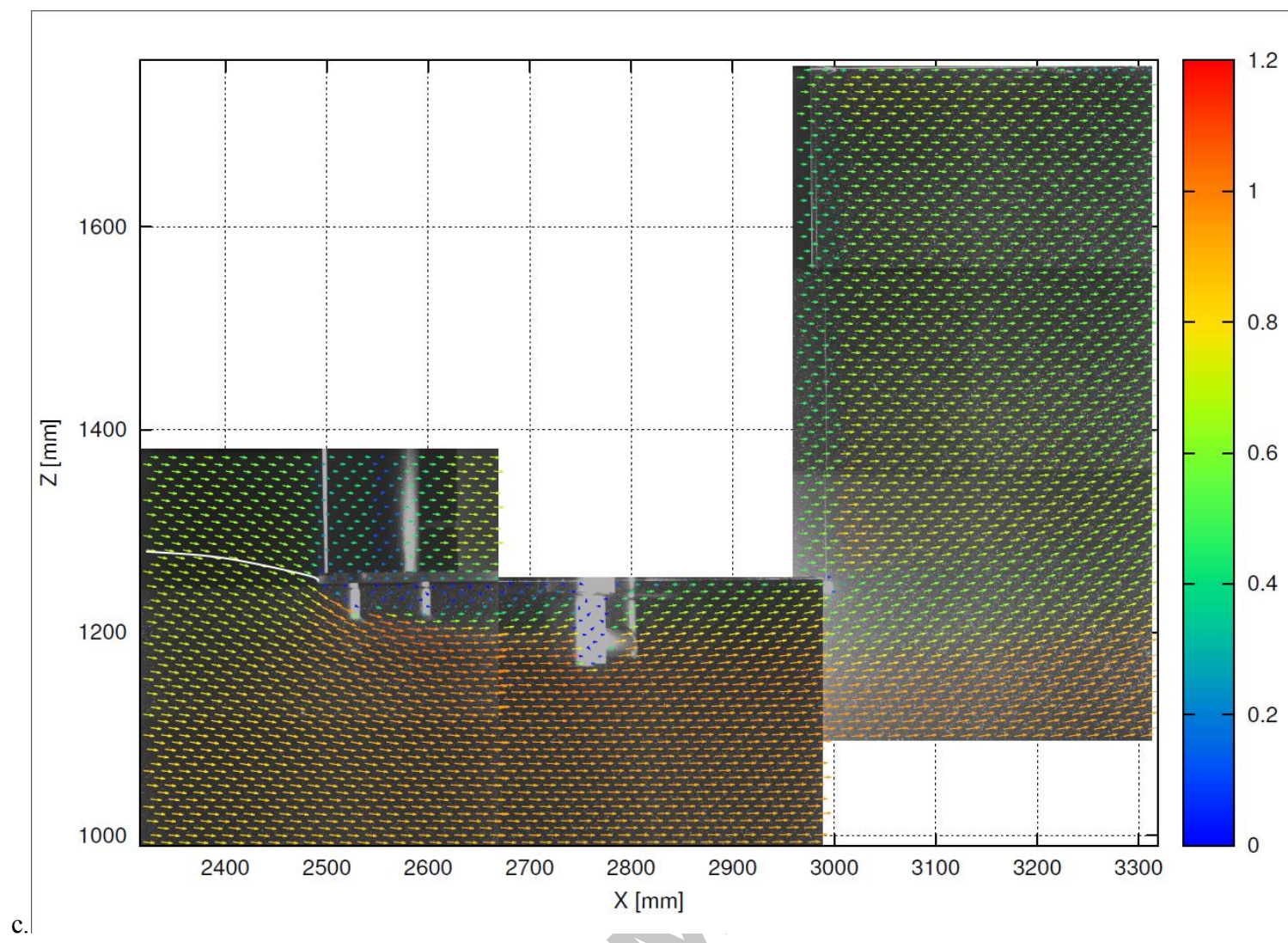

Figure 8: Flow avoidance at a flow speed of $0.8 \mathrm{~m} / \mathrm{s}$ and tip speed ratios: $\lambda=0$ (a), $\lambda=0.3(\mathrm{~b}), \lambda=0.5$ (c).

The PIV data shows that the modelling approach does not simply consist of calibrating the model to yield a single correlating measurement quantity such as the power coefficient. Indeed, the velocity field correlation is of good quality, although some areas were unavoidably shaded from the light source in the experiments. The main flow features are captured by the CFD model, exhibiting an accelerated flow between the blades exposed to the flow and a very effective shielding of the blades on their return stroke. The model also captures the strong inflexion imparted to the flow by the rotor when the turbine is immobilised (Figure 9). This effect reduces as the tip speed ratio increases to 0.3 (Figure 10) and 0.5 (Figure 11).

A detailed review of the results in Figure 9, Figure 10 and Figure 11 shows that the correlation with an immobilised rotor is better than that yielded in the other cases, with a tip speed ratio of 0.3 and 0.5 . The main source of discrepancy is the low velocity region downstream of the housing. The experimental results show a slower rotation than numerically predicted. This may be due to the turbulence handling chosen for the study and it is the subject of a more detailed study focused purely on the CFD model. 


\section{ACCEPTED MANUSCRIPT}
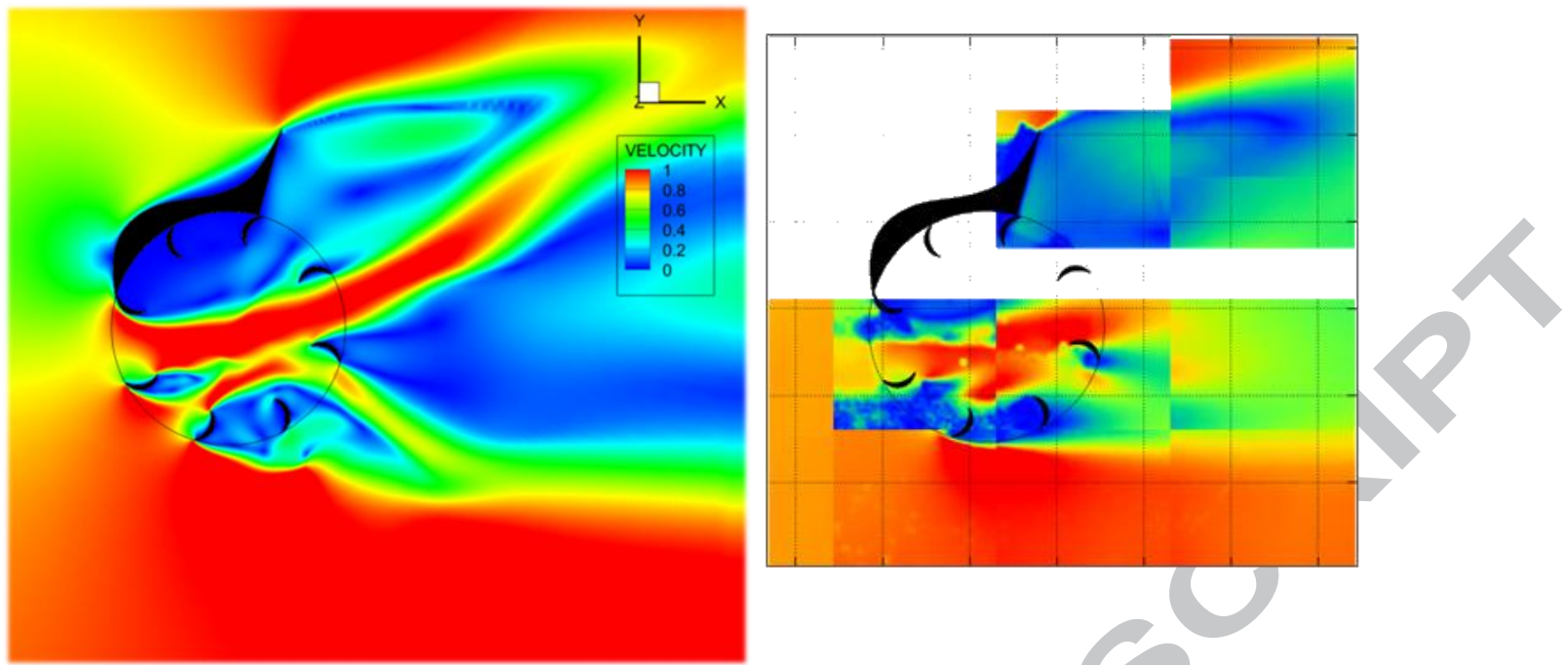

Figure 9: Comparison of numerically predicted velocity contours and PIV - flow speed $0.8 \mathrm{~m} / \mathrm{s}$, TSR 0, common colour scale used.
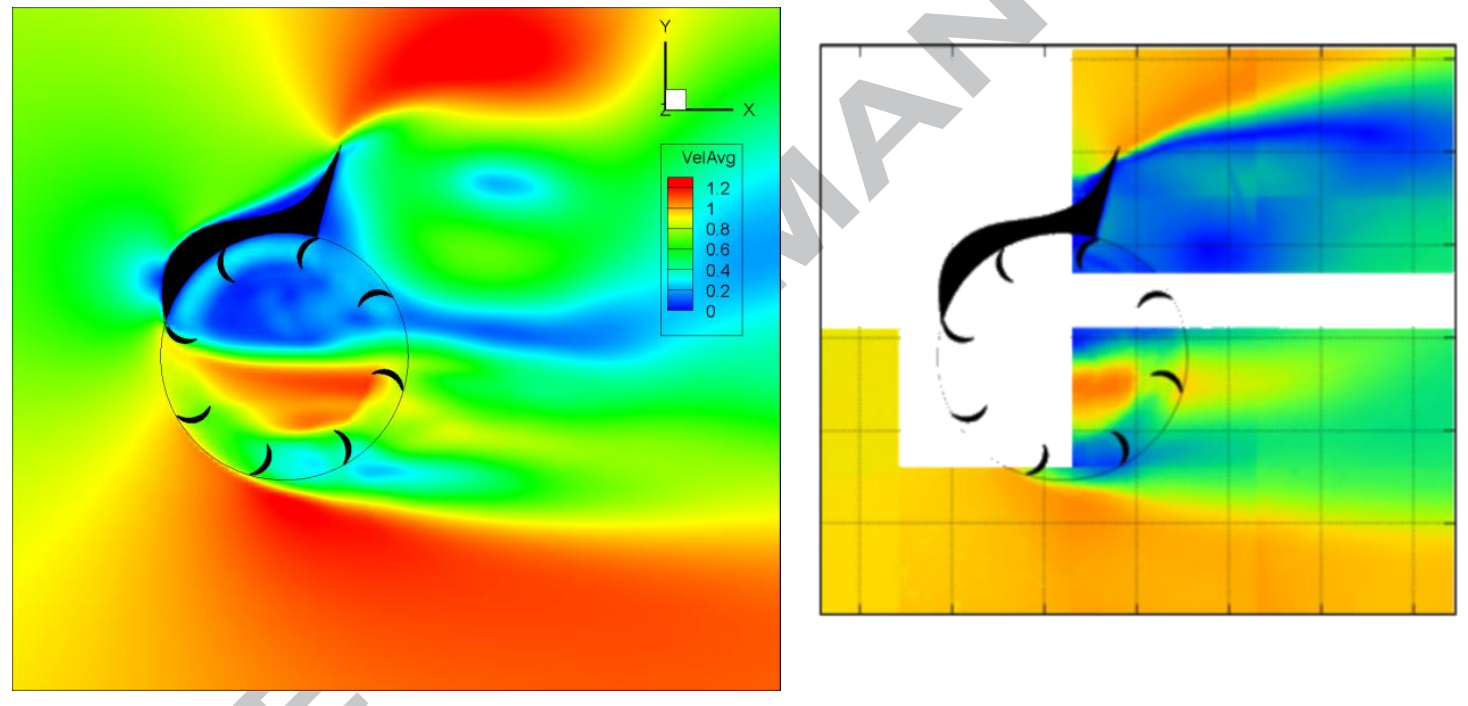

Figure 10: Comparison of numerically predicted velocity contours and PIV - flow speed $0.8 \mathrm{~m} / \mathrm{s}$, TSR 0.3 , common colour scale used.
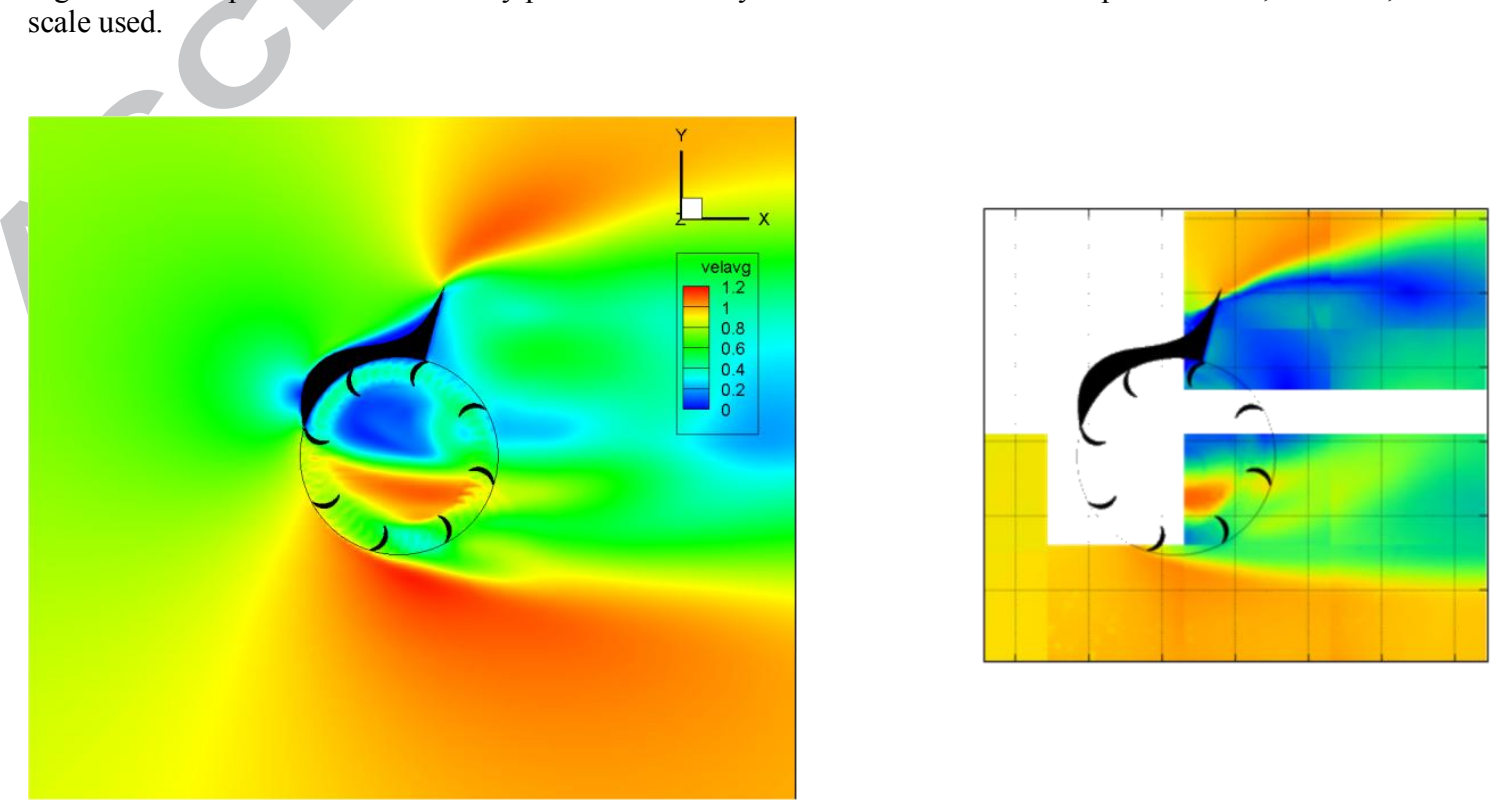
Figure 11: Comparison of numerically predicted velocity contours and PIV measurements - flow speed $0.8 \mathrm{~m} / \mathrm{s}$, TSR 0.5 , common colour scale used.

The analysis of the results gives several indications about the mechanisms through which this turbine operates. The first mechanism is drag: from the shape of the blades and their pitch angle on the rotor, it is instinctive to conclude that the torque contribution from the blades at a south azimuthal position is generated by drag mechanism. This is supported by the pressure contours in Figure 12, which show that high pressure gradients across the blades in the west to south azimuthal quadrant. The flow fast flow through the centre of the turbine combined with the simulated instantaneous torque contributions in Figure 13 indicate that lift has a significant contribution to the torque generation as they continue to generate significant torque levels when reaching an east azimuthal position. For this aspect of the study, a Reynolds number value based on the blade chord may be more relevant. Its range is $R e=$ $4.37 \times 10^{4}$ to $R e=7.65 \times 10^{4}$. This means that the blade is undergoing transition flow regimes. The transition regime has significant implications on the lift and drag properties of blades [21]. Modelling transition regimes and the dynamic stall phenomena occurring around rotating blades is recognised as challenging [5] [6]. This highlights the strength of the simplified approach to turbulence modelling.
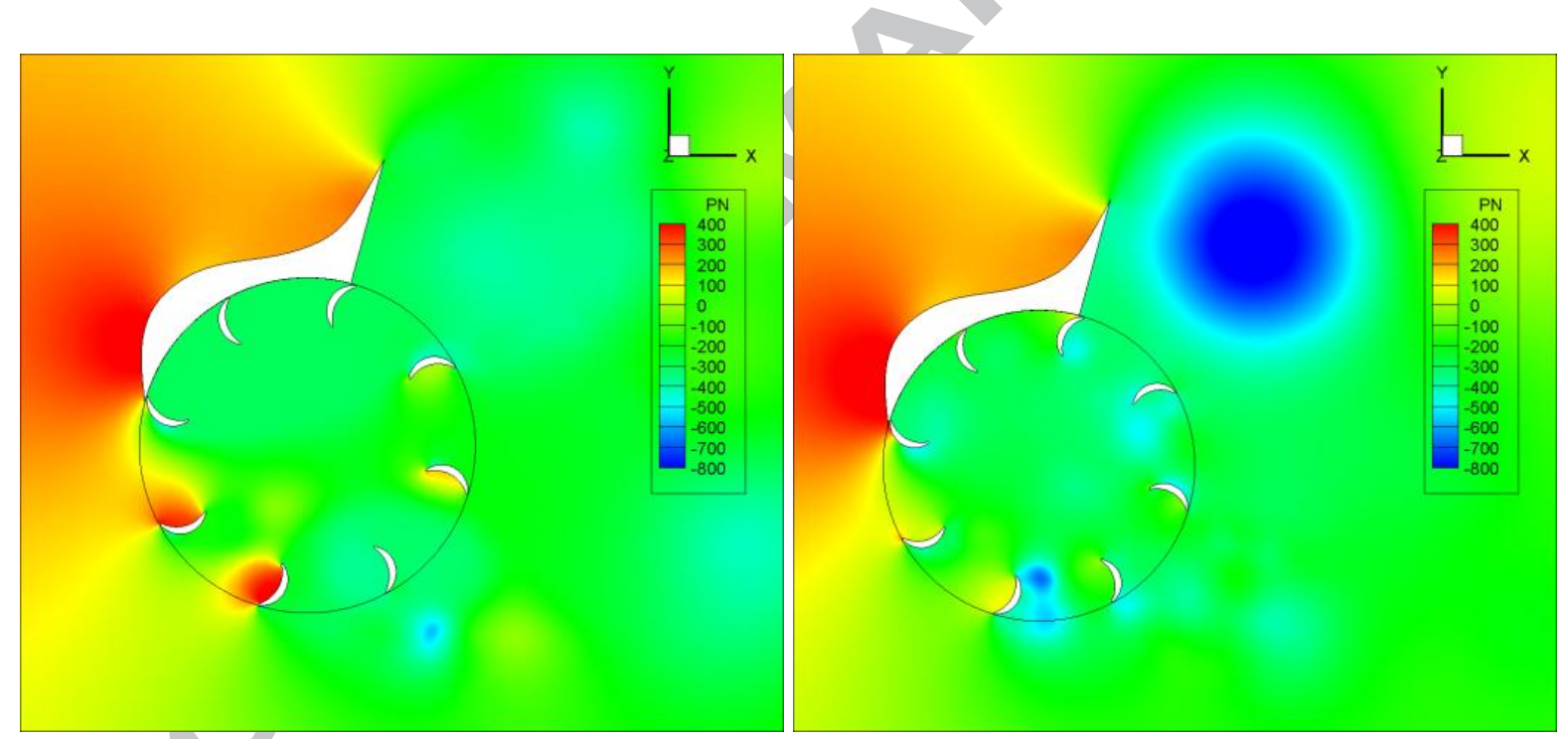

Figure 12: Instantaneous pressure contours from the simulations at $0.8 \mathrm{~m} / \mathrm{s}$, tip speed ratio 0 (left and 0.5 (right)
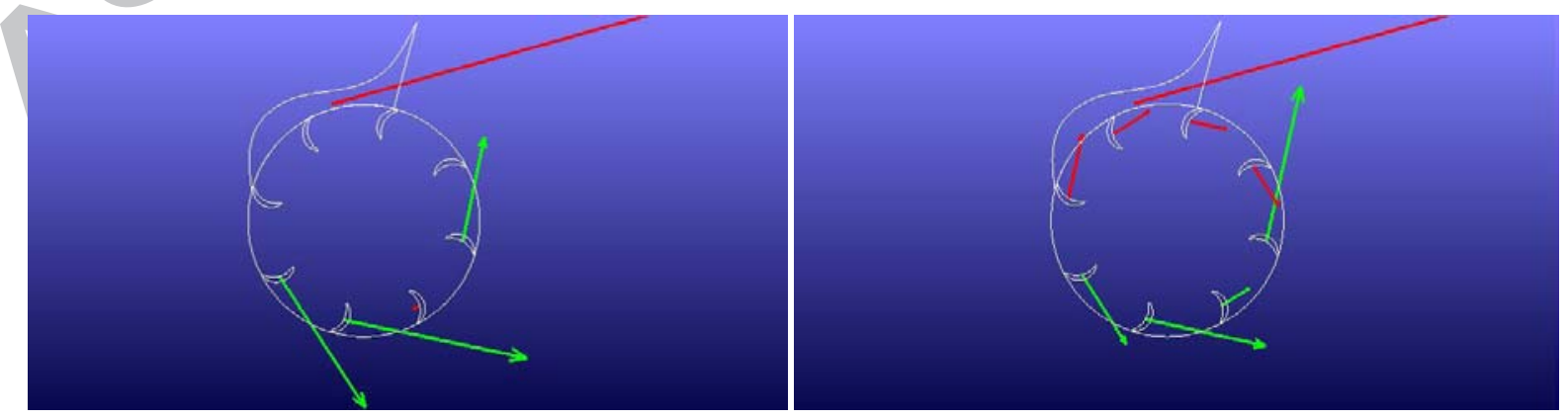

Figure 13: Instantaneous torque contributions from the blades and on the shield at tip speed ratio 0 (left) and 0.5 (right) 


\section{Conclusions}

The work presented shows that the numerical model developed is able to capture the performance of the proposed vertical axis turbine design under test conditions. In particular, the model's response to flow speed captures the increase in efficiency observed with increased flow speed. Importantly, the comparison with the PIV images shows that the modelling approach effectively captures the physics of the flow within the resolution of the experimental data available, rather than simply fit a single criterion such as the power coefficient. Finally, the present work, in conjunction with prior publications demonstrates that a simple approach to turbulence modelling can be an effective and efficient alternative to more complex turbulent models. Combined with the PIV data, it highlights the need for further research in turbulence modelling to cover the broad range of flow regimes encountered and predict correctly their aerodynamic properties.

\section{Acknowledgements}

The authors would like to thank C-FEC Ltd for their sponsorship of the research program, Quadratec Ltd for the design and build of the prototype, and the MARINET European Framework Programme for the access to the IFREMER test facility at Boulogne/Mer, France.

\section{References}

[1] The Crown Estate, "Crown Estate unlocks further UK wave and tidal current opportunities," 8 July 2014. [Online]. Available: http://www.thecrownestate.co.uk/newsand-media/news/2014/further-uk-wave-and-tidal-opportunities-unlocked/. [Accessed October 2014].

[2] C. M. Johnstone, T. McCombes, A. S. Bahaj, L. E. Myers, B. Holmes, J. P. Koefoed and C. Bittencourt, "EquiMar: development of best practices for the engineering performance appraisal of wave and tidal energy convertors," in 9th European Wave and Tidal Energy Conference, Southampton, GB, 2011.

[3] S. Rolland, M. Thatcher, W. Newton, A. J. Williams, T. N. Croft, D. T. Gethin and M. Cross, "Benchmark experiments for simulations of a vertical axis wind turbine," Applied Energy, vol. 111, pp. 1183-1194, 2013.

[4] S. Rolland, W. Newton, A. J. Williams, T. N. Croft, D. T. Gethin and M. Cross, "Simulation technique for the design of a vertical axis wind turbine device with experimental validation," Applied Energy, vol. 111, pp. 1195-1203, 2013.

[5] P. Bachant and M. Wosnik, "Reynolds number dependence of cross-flow turbine performance and near-wake characteristics," in 2nd Marine Energy Technology Symposium, Seattle, USA, 2014.

[6] R. Lanzafame, S. Mauro and M. Messina, "2D CFD Modeling of H-Darrieus Wind 
Turbines Using a Transition Turbulence Model," Energy Procedia, vol. 45, p. 131-140, 2014.

[7] F. R. Menter, “"Two-Equation Eddy-Viscosity Turbulence Models for Engineering Applications," AIAA Journal, vol. 32, no. 8, pp. 1598-1605, 1994.

[8] J. McNaughton, F. Billard and A. Revell, "Turbulence modelling of low Reynolds number flow effects," Journal of Fluids and Structures, vol. 47, pp. 124-138, 2014.

[9] C. Li, S. Zhu, Y.-L. Xu and Y. Xiao, "2.5D large eddy simulation of vertical axis wind turbine in consideration of high angle of attack flow," Renewable Energy, vol. 21, pp. 317-330, 2013.

[10] A. J. Williams, T. N. Croft, I. Masters, C. R. Bennett and M. R. Willis, "A combined BEM-CFD model for tidal stream turbines," in Third international conference on ocean energy, Bilbao, Spain, 2010.

[11] F. Maganga, G. Germain, J. V. Facq, B. Gaurier, E. Rivoalen and G. Pinon, "Caractérisation expérimentale du sillage généré par une hydrolienne - influence du taux de turbulence ambient," in XIèmes Journées Nationales Génie Côtier / Génie Civil, Les Sables d'Olonne, France, 2010.

[12] P. Mycek, B. Gaurier, G. Germain, G. Pinon and E. Rivoalen, "Experimental study of the turbulence intensity effects on marine current turbines behaviour. Part I: One single turbine," Renewable Energy, vol. 66, pp. 729-746, 2014.

[13] A. S. Bahaj, A. F. Molland, J. R. Chaplin and W. M. J. Batten, “ Power and thrust measurements of marine current turbines under various hydrodynamic flow conditions in a cavitation tunnel and a towing tank," Renewable Energy, vol. 32, no. 3, pp. 407-426, 2007.

[14] E. Bouhoubeiny, G. Germain and P. Druault, "Time-Resolved PIV investigations of the flow field around a cod-end net structure," Fisheries Research, vol. 108, no. 2-3, pp. 344-355, 2011.

[15] T. N. Croft, K. Pericleous and M. Cross, "PHYSICA: a multiphysics environment for complex flow processes," in Proceedings of Numerical Methods in Laminar and Turbulent Flow '95, IX, Atlanta, USA, 1995.

[16] P. Chow, M. Cross, K. Pericleous and A. Koulis, "A natural extension of the conventional finite volume method into polygonal unstructured meshes for CFD application," Applied Mathematical Modelling, vol. 20, no. 2, pp. 170-183, 1995.

[17] C. M. Rhie and W. L. Chow, "Numerical study of Turbulent Flow Past and Airfoil with Trailing Edge Separation,” AIAA Journal, vol. 21, pp. 1525-1532, 1983.

[18] S. V. Patankar and D. B. Spalding, "A Calculation Procedure for Heat, Mass and Momentum Transfer in Three-Dimensional Parabolic Flows," International Journal of Heat and Mass Transfer, vol. 15, no. 10, pp. 1787-1806, 1972.

[19] J. P. Van Doormal and G. D. Raithby, "Enhancement of the SIMPLE Method for Predicting Incompressible Fluid Flow," Numerical Heat Transfer, vol. 7, pp. 147-163, 1984. 
[20] F. Hafezi, S. A. Rolland, D. McBride, T. N. Croft, M. Cross and R. Ellis, "A CFD Case Study of Using Turbulence Models for Evaluating the Performance of a Drag-Based Vertical-Axis Wind Turbine," in Proceedings of ACME-UK 2015, 23rd Conference on Computational Mechanics, Swansea, UK, 2015.

[21] I. H. Abbot and A. E. Von Doenhoff, "Chapter 5: The effect of viscosity," in Theory of Wing Sections, Dover Edition, New York, USA, Dover Publications Inc., 1959, pp. 81110. 